Mohamed Tanta, Vítor Monteiro, Bruno Exposto, J. G. Pinto, Antonio P. Martins, Adriano S. Carvalho, Andrés A. Nogueiras Meléndez and João L. Afonso

"Simplified Rail Power Conditioner Based on a Half Bridge Indirect AC/DC/AC Modular Multilevel Converter and a V/V Power Transformer"

In the 43rd Annual Conference of the IEEE Industrial Electronics Society, IECON 2017, Beijing, China, pp.6431-6436, Oct 2017

ISBN: 978-1-5386-1127-2

DOI: 10.1109/IECON.2017.8217120 
Mohamed Tanta, Vítor Monteiro, Bruno Exposto, J. G. Pinto, Antonio P. Martins, Adriano S. Carvalho, Andrés A. Nogueiras Meléndez and João L. Afonso "Simplified Rail Power Conditioner Based on a Half Bridge Indirect AC/DC/AC Modular Multilevel Converter and a V/V Power Transformer". In the 43rd Annual Conference of the IEEE Industrial Electronics Society, IECON 2017, Beijing, China, pp.6431-6436, Oct 2017, ISBN: 978-1-5386-1127-2, DOI: 10.1109/IECON.2017.8217120

\title{
Simplified Rail Power Conditioner Based on a Half-Bridge Indirect AC/DC/AC Modular Multilevel Converter and a V/V Power Transformer
}

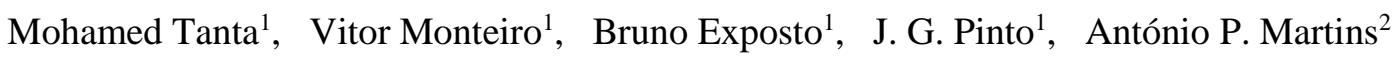 \\ Adriano S. Carvalho ${ }^{2}$, Andrés A. Nogueiras Meléndez ${ }^{3}$ and João L. Afonso ${ }^{1}$ \\ ${ }^{1}$ Centro ALGORITMI - University of Minho, Guimarães - Portugal \\ ${ }^{2}$ SYSTEC Research Center - University of Porto, Porto - Portugal \\ ${ }^{3}$ Departamento de Tecnología Electrónica - University of Vigo, Vigo - Spain \\ ${ }^{1}$ \{mohamed.tanta | vitor.monteiro | bruno.exposto | gabriel.pinto |joao.l.afonso\}@ algoritmi.uminho.pt, ${ }^{2}\{$ ajm $\mid$ asc $\} @$ fe.up.pt, \\ 3 aaugusto@uvigo.es
}

\begin{abstract}
This paper presents a comprehensive study about a Simplified Rail Power Conditioner (SRPC) based on a half-bridge indirect AC/DC/AC Modular Multilevel Converter (MMC) and a V/V traction power transformer. The proposed system with a half-bridge MMC can decrease the costs, reduce the control complexity, and require less hardware devices in comparison with the rail power conditioner based on a full-bridge indirect AC/DC/AC MMC. Moreover, the SRPC with a half-bridge MMC is able to compensate current harmonics, reactive power, and the Negative Sequence Components (NSCs) of currents, which are caused by the unbalance loads between power grid phases. This paper explains the system architecture and its control algorithms based on a pulse-width modulation and a proportional-integral controller, which is used to control the compensation currents. The simulation results of the SRPC show the submodule voltage balancing control and the DC-bus voltage control in order to verify its effectiveness. The compensation strategy based on the NSCs detection is described and evaluated through simulation results.
\end{abstract}

Keywords-Simplified Rail Power Conditioner (SRPC); Power Quality; Electric Locomotive; Harmonics; Modular Multilevel Converter (MMC).

\section{INTRODUCTION}

Traction power supply systems are normally equipped with single-phase traction power transformers to feed the locomotives with the needed electrical power. From the public power grid's point of view, these electric locomotives are huge single-phase loads and they are one of the main causes of public power grid phases unbalance [1]. Consequently, the power quality factor is degraded and the Negative Sequence Components (NSCs) of currents and the harmonics start to appear [2]. To mitigate this, new technologies based on power electronics solutions started to emerge in the markets.

In 1993, Japanese researchers developed the fundamental outlines for the Rail Power Conditioner (RPC) [3] based on two single-phase, two-level, full-bridge, back-to-back converters [2] to solve the problem of NSCs of currents and harmonics. This enabled balanced traction loads without power quality deterioration. The indicated RPC system that integrated two-level converters, as shown in Fig. 1, has some disadvantages in terms of the converter's power rating at the medium voltage levels, besides the applied high switching frequency for power electronics switches. Therefore, two single-phase step-down coupling power transformers were required to connect the RPC with the traction power supply system [2], [4]. However, these step-down coupling power transformers could extremely increase the implementation costs of RPC, besides the required huge space for installation [5].

The RPC based on a Modular Multilevel Converter (MMC) is a relevant solution for the medium voltage applications, where the converter's total power rating is divided equally between several inner two-level converters. Therefore, the power electronics switches can withstand the medium voltage levels [1], [6]. Consequently, the RPC based on MMC can be connected without using step-down coupling transformers. Furthermore, the MMC power switches can operate at a lower switching frequency, which effectively decreases the switching losses [7]. The RPC based on a full-bridge indirect AC/DC/AC $\mathrm{MMC}$ and a V/V power transformer is shown in Fig. 2, and is explained in [1], [6], whereas the same previous referred RPC topology, but integrated with a Scott power transformer is presented in [7]. Both arrangements are equipped with two single-phase, full-bridge, back-to-back, indirect MMC and a DC-link with total four legs. Since the number of hardware devices is related with the required voltage level, implementing the previous arrangement with a high voltage level is expensive [8]. Furthermore, the complexity of MMC control algorithm is proportional to the number of voltage levels.

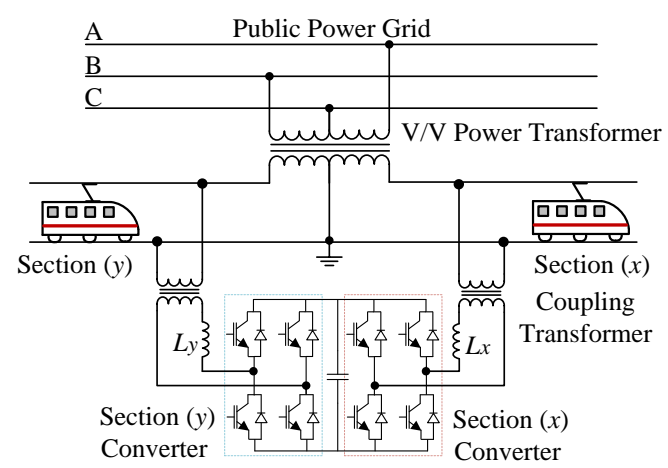

Fig. 1. RPC based on a full-bridge two-level back-to-back converter. 


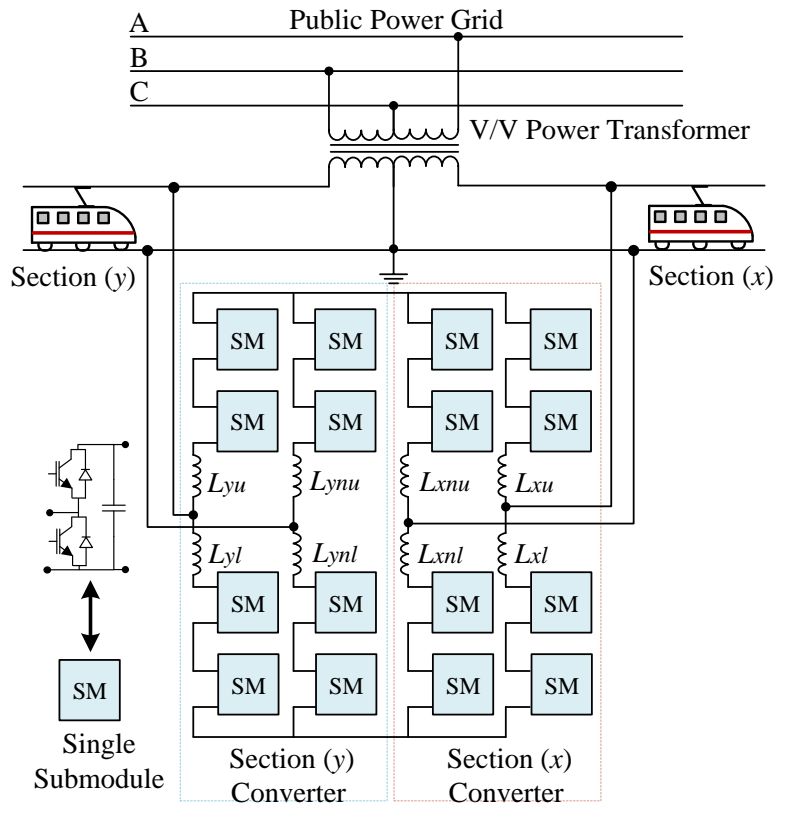

Fig. 2. RPC based on a full-bridge indirect AC/DC/AC MMC.

The aforementioned drawbacks enable the necessity to study a new power conditioner without the use of step-down coupling transformers and with less number of hardware devices. The Simplified Rail Power Conditioner (SRPC) based on a half-bridge indirect AC/DC/AC MMC, shown in Fig. 3, is a convenient solution to improve the power quality of the public power system, which is generally integrated with the traction power supply system. Furthermore, this system has less hardware components, and consequently, it has a lower cost than other RPCs based on MMC [8].

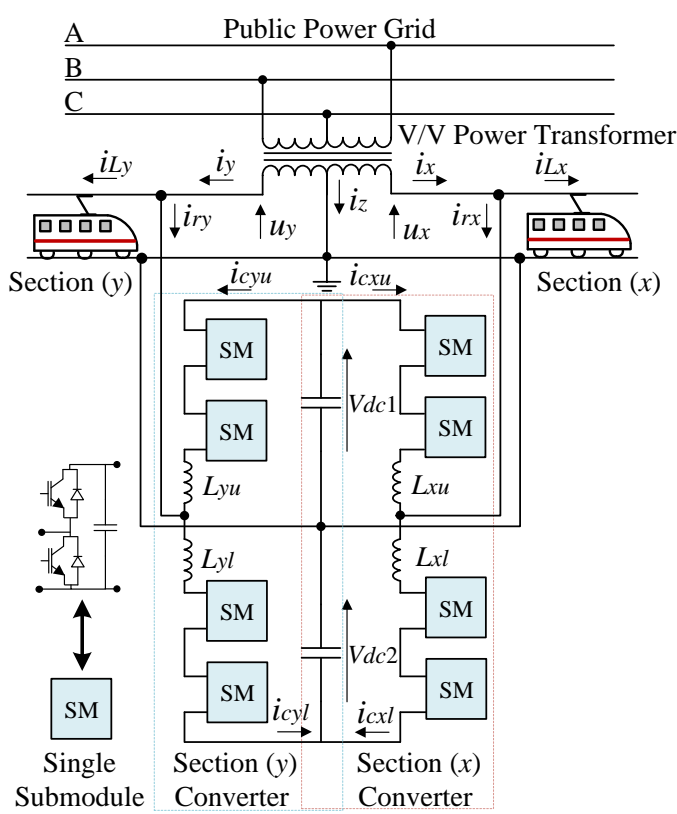

Fig. 3. SRPC based on a half-bridge indirect AC/DC/AC MMC.

The SRPC consists of two half-bridge MMCs connected by two DC-link capacitors in series, as shown in Fig. 3. Each single Submodule (SM) is formed by a half-bridge chopper cell. The output voltage of each SM will be equal to the capacitor's voltage when it is inserted or will be equal to zero when the capacitor is bypassed [9].

This paper presents a study about the SRPC system, and it is organized as follows: Section II describes in detail the SRPC compensation principle and the mathematical analysis, Section III presents the control algorithm of SRPC, Section IV presents the SRPC parameters design, Section V shows the simulation results of one defined case study, to evaluate the performance of the proposed solution. Finally, section VI summarizes the conclusions of the work.

\section{SRPC COMPENSATION PRINCIPLE AND ANALYSIS}

The main objective of the compensation principle is to calculate the compensation current references $i_{r x}{ }^{*}, i_{r y}{ }^{*}$ which the SRPC should inject for both load sections $(x)$ and $(y)$ to compensate the NSCs of currents and harmonics [10]. As in the case of RPC, the SRPC is also able to carry half of the load sections currents difference, from the highly loaded section to the lightly loaded one [3]. As shown in Fig. 3, the voltages at the high voltage power grid side (primary windings of the V/V power transformer) are denoted as $u_{A}, u_{B}, u_{C}$, while the voltages at the traction power grid side (secondary windings of the V/V power transformer) are denoted as $u_{x}, u_{y}$. The phase $u_{z}$ is grounded and connected to the center-split midpoint of the DC-bus. The currents of both load sections are denoted as $i_{L x}$, $i_{L y}$, while the MMC leg currents are denoted as $i_{c x}, i_{c y}$.

For a normal operating case, and without applying a compensation method $\left(i_{r x}=i_{r y}=0\right)$, the traction power grid currents are given by the following equations [1], [2]:

$$
\left[\begin{array}{c}
\dot{I}_{x} \\
\dot{I}_{y} \\
\dot{I}_{z}
\end{array}\right]=\left[\begin{array}{c}
I_{L x} \angle-30^{\circ} \\
I_{L y} \angle-90^{\circ} \\
-I_{L x} \angle-30^{\circ}-I_{L y} \angle-90^{\circ}
\end{array}\right]=\left[\begin{array}{c}
I_{x} \angle-30^{\circ} \\
I_{y} \angle-90^{\circ} \\
-I_{x} \angle-30^{\circ}-I_{y} \angle-90^{\circ}
\end{array}\right]
$$

With the purpose of making the three-phase power grid currents balanced, it is important to add a certain active power component to the lightly loaded section and to subtract the same active power amount from the highly loaded section [11]. In this case, $\dot{I}_{x}, \dot{I}_{y}$ have the same magnitudes but $\dot{I}_{z}$ has a different one because the angles between the currents are not equal to $120^{\circ}$. However, the currents are still imbalanced and a certain reactive power component should be generated by the highly loaded section (the highly loaded section converter compensates a quantity of a capacitive reactive current) to be delivered to the lightly loaded one (the lightly loaded section converter compensates a quantity of an inductive reactive current) [2], [3]. As a result, and after compensating both of the active and the reactive powers, the three-phase power grid currents are balanced. The active current component which is added/subtracted to the lightly/highly loaded section:

$$
I_{r x a}=I_{r y a}=\frac{1}{2} \Delta I_{L}
$$

The capacitive reactive current which is injected by the highly loaded section converter has the same magnitude value of the inductive reactive current which is injected by the lightly loaded section converter as shown in equation (3) [12]. 


$$
I_{r x r}=I_{r y r}=\frac{1}{2}\left(I_{L x}+I_{L y}\right) \tan \frac{\pi}{6}
$$

Phase $x$ and phase $y$ currents after compensation are:

$$
\begin{gathered}
\dot{I}_{x x}=\dot{I}_{L x}+\dot{I}_{r x a}+\dot{I}_{r x r}=\frac{1}{\sqrt{3}}\left(I_{L x}+I_{L y}\right) \angle 0^{\circ} \\
\dot{I}_{y y}=\dot{I}_{L y}+\dot{I}_{r y a}+\dot{I}_{r y r}=\frac{1}{\sqrt{3}}\left(I_{L x}+I_{L y}\right) \angle 120^{\circ} \\
\dot{I}_{z z}=-\dot{I}_{y y}-\dot{I}_{x x}
\end{gathered}
$$

Fig. 4 shows the phasor diagrams after compensation, to clarify the previous mathematical equations, after assuming a unity power factor for both load sections and neglecting the power losses in SRPC power electronics devices. Fig. 4(b) shows the currents on the secondary of the V/V power transformer, after assuming the section $(x)$ as a highly loaded section and section $(y)$ as a lightly loaded section.

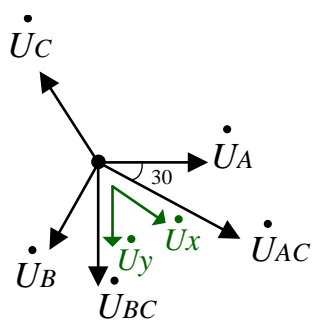

(a)

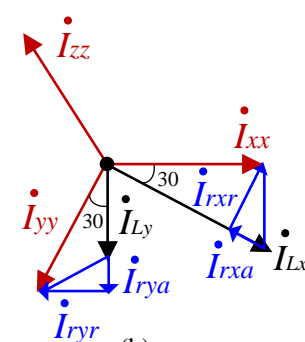

(b)
Fig. 4. Phasor diagrams after compensation: (a) V/V transformer primary and secondary voltages; (b) Currents on the secondary side of a V/V transformer.

\section{CONTROL ALGORITHM OF SRPC}

\section{A. Compensation Currents Reference Signals Generation}

One of the main objectives of SRPC control system is to calculate the final compensation currents references of $i_{r x}{ }^{*}, i_{r y}{ }^{*}$ [13]. From the Fig. 4(b), it is possible to write equations (5).

$$
\begin{aligned}
& i_{r x}^{*}=i_{x x}-i_{L x} \\
& i_{r y}^{*}=i_{y y}-i_{L y}
\end{aligned}
$$

Using two current sensors on both load sections of $(x)$ and (y) can be useful to acquire both of $i_{L x}$ and $i_{L y}$. In order to get the reference currents signals, the traction power system currents after compensation $i_{x x}$ and $i_{y y}$ should be calculated as in equations (4). This is possible by applying the control strategy that presented in Fig. 5. The load sections currents $i_{L x}$ and $i_{L y}$ are the most important variables because they have considered as the input signals for this control system to calculate the compensation currents references. The locomotives are normally connected with power converters to drive the traction motors. Therefore, it is possible to consider a unity load power factor. Consequently, both load sections currents only have the active current components $i_{L x a}, i_{L y a}$ and the harmonics contents $i_{L x h}, i_{L y h}$ [2], then the instantaneous values are as in (6):

$$
\begin{aligned}
& i_{L x}=\sqrt{2} I_{L x a} \sin \left(\omega t-\frac{\pi}{6}\right)+\sum_{h=2}^{\infty} i_{L x h} \\
& i_{L y}=\sqrt{2} I_{L y a} \sin \left(\omega t-\frac{\pi}{2}\right)+\sum_{h=2}^{\infty} i_{L y h}
\end{aligned}
$$

Multiplying the signal of $i_{L x}$ by $\sin (\omega t-\pi / 6)$ and the signal of $i_{L y}$ by $\sin (\omega t-\pi / 2)$, then summing the obtained results, gives a DC current component $I_{P}$ as in equation (7). The correspondent sine waves are obtained by using an enhanced linear Phase-Locked Loop (PLL) [14].

$$
I_{P}=\frac{\sqrt{2}}{2}\left(I_{L x a}+I_{L y a}\right)
$$

It is important to use a low pass filter (LPF) to obtain the $I_{P}$ signal without any harmonics [11]. From (2) and Fig. 4(b), the currents values after only shifting the active power difference from the highly loaded section to the lightly loaded one are:

$$
\begin{aligned}
& \dot{I}_{x I}=\dot{I}_{L x}+\dot{I}_{r x a}=\frac{1}{2}\left(I_{L x}+I_{L y}\right) \angle-30^{\circ} \\
& \dot{I}_{y 1}=\dot{I}_{L y}+\dot{I}_{r y a}=\frac{1}{2}\left(I_{L x}+I_{L y}\right) \angle-90^{\circ}
\end{aligned}
$$

The next step is to calculate the instantaneous currents values after only shifting the active power difference $i_{x 1}, i_{y 1}$. This is possible by multiplying the value of $I_{P} / \sqrt{2}$ with the correspondent sine waves. The same is applicable for (3) to calculate the instantaneous values of $i_{r x r}, i_{r y r}$ after multiplying the signals with the correspondent shifted sine waves of $\sin (\omega t+\pi / 3)$ and $\sin (\omega t-\pi)$. The shifter in Fig. 5 is mainly responsible to acquire the shifted sine waves [3]. Finally, the equations (4) are calculated by the compensation strategy presented in Fig. 5.

\section{B. Modulation Technique, DC-bus Voltage Balancing Control and MMC SMs Voltage Control}

This section presents some information about the modulation technique that is used to drive the power electronics switches (IGBTs), DC-bus capacitors voltage balancing control and MMC SMs voltage control to guarantee a good compensation performance. Taking into account that the DC-bus voltage is applied in two capacitors placed in series, voltage balancing control for these capacitors is absolutely necessary to maintain equal voltage values for the upper and the lower arms of the SRPC. Otherwise, if two DC-bus capacitors voltages are unbalanced, then it could cause voltage fluctuations and current distortion [3], [8]. Two Proportional-Integral (PI) controllers are responsible to achieve the voltage balancing process for the DC-bus capacitors as shown in the control strategy of Fig. 6. One of the PI controllers is used to compare the actual value of DC-bus capacitors with the reference value, then the PI output signal is corrected by multiplying with the correspondent sine waves. The second PI controller is mainly used to achieve a DC-bus voltage balance between capacitors, where the input signal is $\Delta V=V_{d c 2}-V_{d c 1}$. The output signal of this controller $\Delta I_{\text {out }}$ is subtracted/added to the compensation currents reference $i_{r x}{ }^{*}$ and $i_{r y}{ }^{*}$ respectively. When the control system is at the half positive cycle of $i_{r \sigma}^{*}, \sigma \in\{x, y\}$, the second PI controller will output a positive offset $\Delta V>0 \Rightarrow V_{d c 2}>V_{d c 1}$ in order to make the reference current of $i_{r y}{ }^{*}$ increase to $\left|i_{r y}{ }^{*}+\Delta I_{\text {out }}\right|$. As a result, the capacitor $\mathrm{C}_{2}$ would discharge with more time in the control cycle to make the current increase, so the voltage of capacitor $C_{2}$ would 


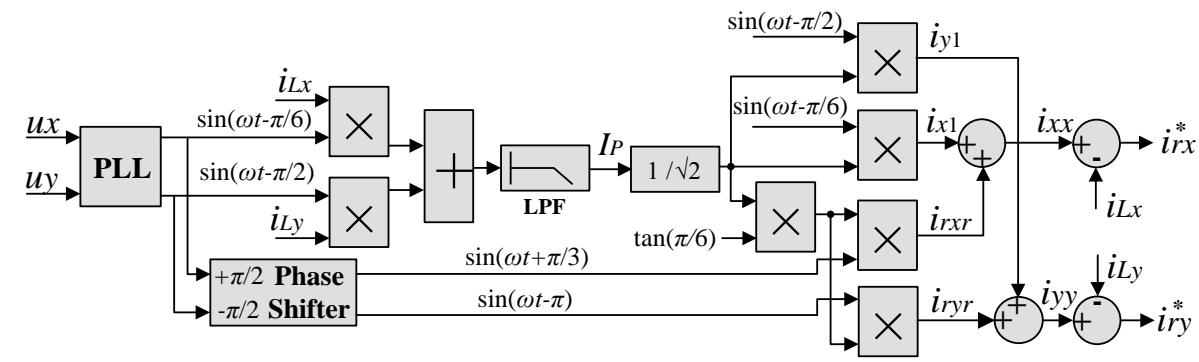

Fig. 5. SRPC control strategy to calculate the reference signals in order to compensate the NSCs and harmonics.

decrease to be equal to $V_{d c 1}$. In the same time, $\Delta I_{\text {out }}$ signal is subtracted from the reference current signal of phase $x$, then the value of $i_{r x}{ }^{*}$ is decreasing to a value of $\left|i_{r x}^{*}+\Delta I_{\text {out }}\right|$. As a result, $C_{1}$ would charge with more time in the control cycle to make the current decrease, so the voltage of capacitor $C_{1}$ would increase to be equal to $V_{d c 2}$ [3]. The previous principle is also applicable at the half negative cycle of $i_{r \sigma}^{*}, \sigma \in\{x, y\}$. The final reference signals are compared with the actual compensation currents of $i_{r x}, i_{r y}$. Then, PI controllers are used to generate the voltage reference signals $v_{x}{ }^{*}, v_{y}{ }^{*}$, which will be used in the Pulse-Width Modulation (PWM) to drive the power switches as shown in the diagram of Fig. 6. PWM technique has a fixed switching frequency and low current ripples in comparison with other modulation techniques [8].

It is worth to mention that, the DC-bus voltage can be controlled by only one load section converter regardless the other converter when the SRPC is designed to operate with only one loaded section as in [8]. In this case, only one load section is loaded and its converter is responsible to compensate the active, capacitive reactive and harmonics components of currents, while the other converter (unloaded section converter) is responsible to absorb the active power and to compensate inductive reactive current. Then, the last converter is mainly controlled for a fundamental frequency power conditioning and it can handle alone the balance of the DC-bus capacitors [8]. The voltage balancing control for the SMs capacitors is shown in Fig. 7(a). By taking into consideration eight SMs in each MMC leg $2 N=8$, so the total SMs number will be divided equally between the upper and the lower arms in the same leg, then the arm will consist of four SMs $N=4$. Consequently, the PWM triangular carriers are shifted by $360 / N$ degrees between each other. The voltage balancing controller of the SMs capacitors is responsible to adjust every SM capacitor voltage to its reference value. The control is performed by using a proportional controller for each SM to act only dynamically in the balancing process in every switching period. The output signal of this controller is multiplied by +1 when the current direction of $i_{c x}$ is to charge the SM capacitor or is multiplied by -1 if its direction is to discharge the capacitor [9], [15].

Fig. 7(b) shows the averaging control and the circulating current controller for the MMC legs. The averaging control is implemented by summing the actual values for all SMs capacitors, then it compares the average of this actual value with a reference value by using a PI controller. The output of this controller is considered as a reference value for a circulating current controller. The last is implemented by summing the leg currents values $i_{c \sigma u}, i_{c \sigma l} ; \sigma \in\{x, y\}$ in the upper and the lower arms for each load section converter. Then, the final averaging control signals of $A_{\sigma} ; \sigma \in\{x, y\}$ and the voltage reference signals of $v_{\sigma}{ }^{*} ; \sigma \in\{x, y\}$ were added to the obtained voltage balancing control output signals to be compared with the PWM carriers [16].

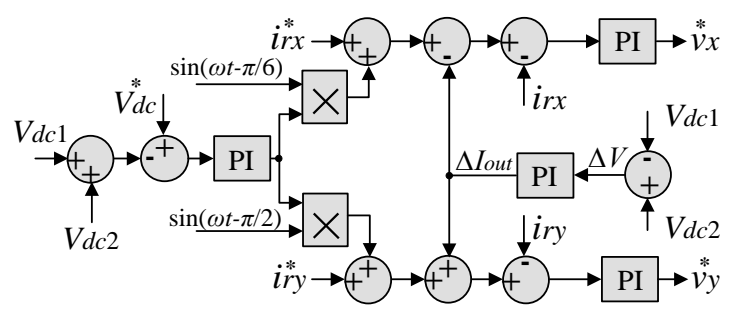

Fig. 6. Voltage balancing control for the DC-bus capacitors.

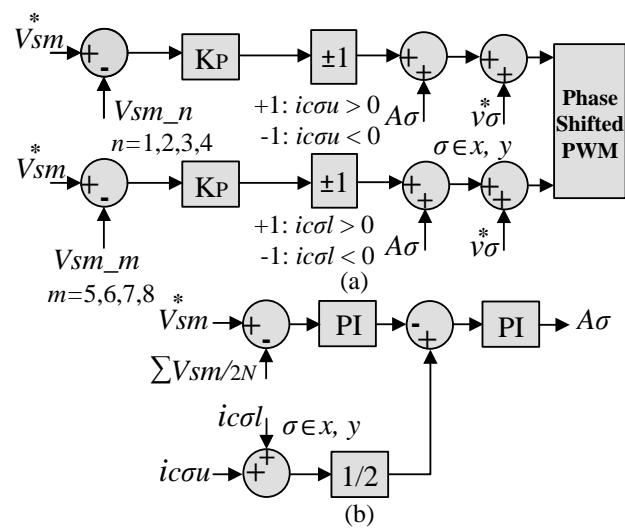

Fig. 7. MMC SMs voltage control; (a) Voltage balancing control for the SMs capacitors; (b) Averaging control and circulating current control.

\section{MMC PARAMETERS DESIGN}

The buffer inductances between MMC arms, SMs capacitors and DC-bus capacitors should have appropriate values to ensure a good compensating performance. This section presents some information related to MMC parameters design. The SRPC with a half-bridge MMC is shown in Fig. 3.

\section{A. MMC Buffer Inductance}

The buffer inductance influences directly in the control system capability to track the compensation current references. It could be designed by considering the factors of current ripples suppression and the signal tracking speed [17]. According to [8], [18], The equation which determines the value of the buffer inductance $L_{\sigma(u, l)} ; \sigma \in\{x, y\}$ is: 


$$
L_{\sigma(u, l)} \leq \frac{\sqrt{\frac{V_{d c}{ }^{2}}{4}-V_{S M}{ }^{2}}}{2 \pi f I_{r \sigma}}
$$

Where $V_{d c}$ is the DC-bus voltage, $V_{S M}$ is the SM capacitor DC voltage, $f$ is the traction power grid frequency, $I_{r \sigma} ; \sigma \in\{x, y\}$ is the half-bridge MMC compensating current.

\section{B. SM Capacitor and DC-bus Capacitors Design}

Smoothing the voltage fluctuations of SMs normally corresponds to a large capacitance of SMs capacitors. However, a large capacitance could increase the cost of SRPC, besides the necessity for an extra size of the final SRPC. The previous principle is considered when selecting the SM capacitor value. Furthermore, the resonance frequency that related to the buffer inductance and the SM capacitor is also considered in SM capacitor design. According to [19], the equation that calculates the minimum capacitance of each SM capacitor is:

$$
C_{S M}>\frac{3 N+2 N m^{2}}{48(2 \pi f)^{2} L_{\sigma(u, l)}}
$$

The modulation index $m \leq 1$ could be calculated from the equation (11). DC-bus capacitor value is recommended to be $N$ times the value of a single SM capacitor.

$$
m=\frac{2 U_{\sigma(p e a k)}}{V_{d c 1}+V_{d c 2}} ; \sigma \in\{x, y\}
$$

\section{SiMULATION RESULTS}

SRPC simulation model has been built by using the PSIM software in order to determine SRPC ability in terms of NSCs and harmonics compensation. The model is determined by $(N+1)$ levels half-bridge MMC with a total number of SMs in each leg $2 N=8$. The voltage values are given by TABLE I. These values are important to estimate the MMC parameters, which have been described in the section IV.

TABLE I: Voltage VALUES USED IN THE Simulation MOdEL

\begin{tabular}{ccc}
\hline \hline Voltage & Symbol & Value \\
\hline \hline Power grid voltage & $U_{A B}$ & $20 \mathrm{kV}$ \\
Traction grid voltage & $U_{\sigma} ; \sigma \in\{x, y\}$ & $1 \mathrm{kV}$ \\
MMC DC-bus & $V_{d c}=V_{d c 1}+V_{d c 2}$ & $3.8 \mathrm{kV}$ \\
MMC SM & $V_{S M}$ & $900 \mathrm{~V}$ \\
\hline \hline
\end{tabular}

From equation (11) and after considering a modulation index value $m$ equals to 0.75 , the MMC DC-bus voltage has the value of $3.8 \mathrm{kV}$ and it is divided between $N$ SMs. Considering the voltage drop across the arm buffer inductance, MMC SM voltage has the value around $900 \mathrm{~V}$. Buffer inductance and SM capacitor values could be estimated from equation (9) and (10) respectively. Then, the SRPC model parameters have the values in TABLE II. Because the load power factor is considered to be one, the locomotives are modeled as a resistive load connected to the output of an uncontrolled full-bridge rectifier [2]. This assumption is important since the rectifier is considered as a harmonics source. The simulation results show one case study when both load sections $(x)$ and $(y)$ were loaded unequally. By assuming the load sections of $(x)$ and $(y)$ are loaded with $1 \mathrm{MW}$, $500 \mathrm{~kW}$ respectively, the public grid currents before compensation are shown in Fig. 8(a). The currents are unbalanced and contain large amounts of NSCs besides the harmonics. In accordance with the case study condition, phase $A$ current has a double value of phase $B$ current, then according to (1), phase $C$ current is resulting by summing the waveforms of other phases $A$ and $B$. Fig. 8(b) shows the same currents after turning on the SRPC. The results indicate balanced currents without NSCs and with less harmonics contents.

TABLE II: SRPC SIMULATION MODEL PARAMETERS

\begin{tabular}{ccc}
\hline Parameter & Symbol & Value \\
\hline \hline Total rated power & $P_{\text {Total }}$ & $1.6 \mathrm{MW}$ \\
Rated frequency & $f$ & $50 \mathrm{~Hz}$ \\
PWM carrier frequency & $f_{c}$ & $2 \mathrm{kHz}$ \\
Equivalent switching frequency & $f_{e q}=N f_{c}$ & $8 \mathrm{kHz}$ \\
Buffer inductance & $L_{\sigma(u, l)} ; \sigma \in\{x, y\}$ & $1 \mathrm{mH}$ \\
SM capacitor & $C_{S M}$ & $10 \mathrm{mF}$ \\
DC-bus Capacitor & $C_{d c}$ & $40 \mathrm{mF}$ \\
\hline \hline
\end{tabular}

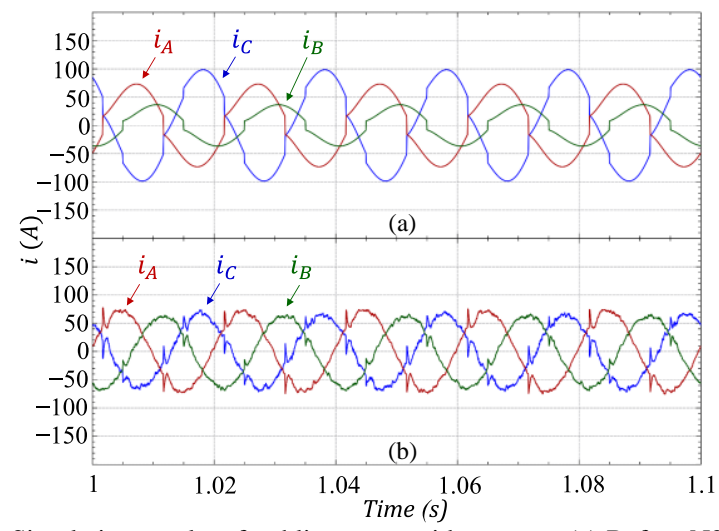

Fig. 8. Simulation results of public power grid currents: (a) Before NSCs and harmonics compensation; (b) After NSCs and harmonics compensation.

Fig. 9(a) presents both load sections currents of $i_{L x}$ and $i_{L y}$. These currents are the main source of NSCs and harmonics in the system because of using uncontrolled rectifiers on both load sections. The compensation currents that injected to the public power grid and generated by the SRPC are shown in Fig. 9(b). Section $(x)$ compensation current generates a capacitive reactive power, while section (y) compensation current generates an inductive reactive power. The effectiveness of the voltage balancing control for each SM capacitor, averaging voltage control for each MMC leg capacitors, the circulating leg currents control in the arms of each MMC leg and the DC-bus capacitors voltage control are shown in the Fig. 10.

Fig. 10(a) presents the SMs voltages of converter $(x)$, where $v_{c x n ; n(1,2,3,4)}$ indicates the upper arm SMs voltages of converter $(x)$ and $v_{c x m ; m(5,6,7,8)}$ indicates the lower arm SMs voltages of converter $(x)$. SMs voltages instantaneous values follow the reference value $V_{S M}$ that indicated in TABLE I. The same is applicable for converter (y) SMs voltages $v_{c y n ; n(1,2,3,4)}$ and $v_{c y m ;} m(5,6,7,7)$ in Fig. 10(b). The fluctuations in the waveforms are normally caused by the $\mathrm{AC}$ components of the circulating current between MMC arms, besides the AC components of the compensation currents. Fig. 10(c) demonstrates the DC-bus capacitors voltage, where the total DC-bus voltage $V_{d c}$ in any cycle is calculated by summing both waveforms of $V_{d c 1}$ and 
$V_{d c 2}$. The reference signal for each DC-bus capacitor is $1900 \mathrm{~V}$, then the total DC-bus voltage is around $3.8 \mathrm{kV}$.

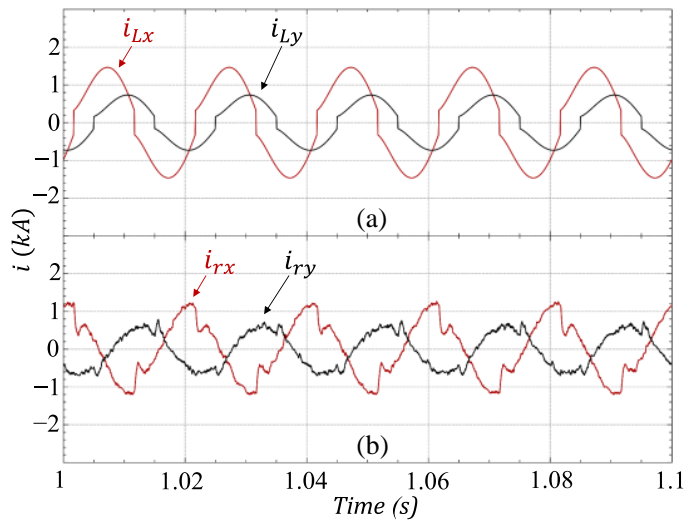

Fig. 9. Simulation results: (a) Load sections currents when both load sections were loaded unequally; (b) Compensation currents provided by the SRPC.

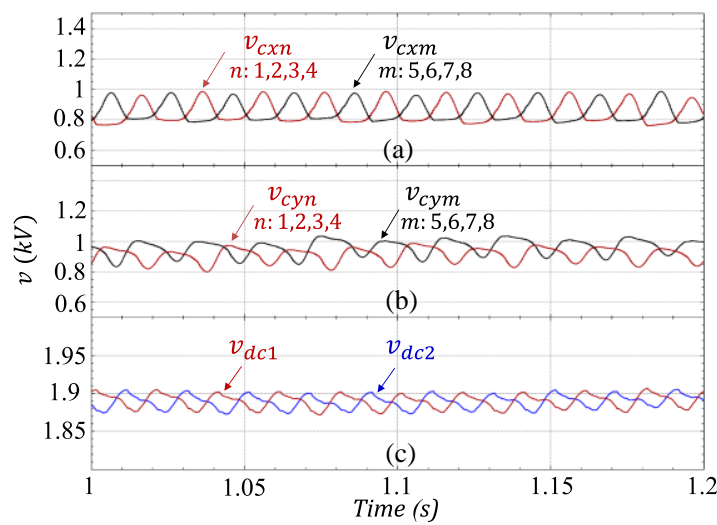

Fig. 10. DC capacitors voltages: (a) SMs voltages of section $(x)$ converter; (b) SMs voltages of section $(y)$ converter; (c) DC-bus capacitors voltages.

\section{CONCLUSION}

This paper discussed a simplified rail power conditioner (SRPC) based on a half-bridge modular multilevel converter (MMC) to compensate the current harmonics and the negative sequence components (NSCs) of currents. In comparison with the rail power conditioner based on a full-bridge MMC, this system can reduce the costs and the hardware complexity. The proposed control strategy ensures a balanced voltage between DC-bus capacitors, besides the ability to achieve balancing voltage control between MMC submodules. A pulse-width modulation technique is used to drive the power switches. The parameters design of half-bridge MMC have also been considered in the literature to build the final simulation model of SRPC. One case study has been considered, in which both load sections were loaded unequally to test the final simulation model with the proposed control strategy. The SRPC represents a new effort to improve the power quality of electrified railways with less complexity and with fewer hardware devices.

\section{ACKNOWLEDGMENT}

Mohamed Tanta was supported by FCT (Fundação para a Ciência e Tecnologia) PhD grant with a reference PD/BD/127815/2016. This work has been supported by COMPETE: POCI-01-0145-FEDER-007043 and FCT within the Project Scope: UID/CEC/00319/2013.

\section{REFERENCES}

[1] M. Tanta, J. A. Afonso, A. P. Martins, A. S. Carvalho, and J. L. Afonso, "Rail Power Conditioner Based on Indirect AC/DC/AC Modular Multilevel Converter Using a Three-phase V/V Power Transformer," in World Congress on Engineering Conference WCE, pp.289-294, July, 2017.

[2] A. Luo, C. Wu, J. Shen, Z. Shuai, and F. Ma, "Railway Static Power Conditioners for High-speed Train Traction Power Supply Systems Using Three-phase V/V Transformers," IEEE Trans. Power Electron, vol.26, no.10, pp.2844-2856, Oct. 2011.

[3] F. Ma, A. Luo, X. Xu, H. Xiao, C. Wu, and W. Wang, "A Simplified Power Conditioner Based on Half-Bridge Converter for High-Speed Railway System," IEEE Trans. Ind. Electron, vol.60, no.2, pp.728-738, Feb. 2013.

[4] I. Perin, P. F. Nussey, T. V. Tran, U. M. Cella, and G. R. Walker, "Rail power conditioner technology in Australian Heavy Haul Railway: A case study," in 2015 IEEE PES Asia-Pacific Power and Energy Engineering Conference (APPEEC), 2015, pp.1-5.

[5] F. Ma, Z. He, Q. Xu, A. Luo, L. Zhou, and M. Li, "Multilevel Power Conditioner and its Model Predictive Control for Railway Traction System," IEEE Trans. Ind. Electron, vol.63, no.11, pp.72757285, Nov. 2016

[6] M. Tanta, V. Monteiro, J. G. Pinto, A. P. Martins, A. S. Carvalho, and J. L. Afonso, "Efficiency and Cost Estimation for a Static Frequency Converter and a Rail Power Conditioner Based on an Indirect Modular Multilevel Converter in Railways Applications," in ICEE $3^{\text {rd }}$ International Conference on Energy and Environment 2017, pp.313-319.

[7] S. Song, J. Liu, S. Ouyang, and X. Chen, "A modular multilevel converter based Railway Power Conditioner for power balance and harmonic compensation in Scott railway traction system," in IEEE 8th International Power Electronics and Motion Control Conference, 2016, pp. 2412-2416.

[8] L. Liu and N. Dai, "Hybrid railway power conditioner based on half-bridge modular multilevel converter," in 2016 IEEE Energy Conversion Congress and Exposition (ECCE), 2016, pp. 1-7.

[9] M. Hagiwara and H. Akagi, "Control and Experiment of PulsewidthModulated Modular Multilevel Converters," IEEE Trans. Power Electron., vol. 24, no. 7, pp. 1737-1746, Jul. 2009.

[10] A. M. Bozorgi, M. S. Chayjani, R. M. Nejad, and M. Monfared, "Improved grid voltage sensorless control strategy for railway power conditioners," IET Power Electron., vol. 8, no. 12, pp. 2454-2461, 2015.

[11]C. Wu, A. Luo, J. Shen, F. J. Ma, and S. Peng, "A Negative Sequence Compensation Method Based on a Two-Phase Three-Wire Converter for a High-Speed Railway Traction Power Supply System," IEEE Trans. Power Electron., vol. 27, no. 2, pp. 706-717, Feb. 2012.

[12] B. Chen, C. Zhang, W. Zeng, C. Tian, and J. Yuan, “An electrical-magnetic hybrid power quality compensation strategy for V/V traction power supply system," in 2014 IEEE Energy Conversion Congress and Exposition (ECCE), 2014, pp. 3774-3779.

[13] V. P. Joseph and J. Thomas, "Power quality improvement of AC railway traction using railway static power conditioner a comparative study," in 2014 International Conference on Power Signals Control and Computations (EPSCICON), 2014, pp. 1-6.

[14]M. Karimi-Ghartemani, "Linear and Pseudolinear Enhanced PhasedLocked Loop (EPLL) Structures," IEEE Trans. Ind. Electron., vol. 61, no. 3, pp. 1464-1474, Mar. 2014.

[15] M. Rejas et al., "Performance comparison of phase shifted PWM and sorting method for modular multilevel converters," in 2015 17th European Conference on Power Electronics and Applications, 2015, pp. 1-10.

[16] G. Liu, Q. Jiang, and Y. Wei, "Study on Capacitor Voltage Balancing Control of Modular Multilevel Converter at Low Frequency," Int. J. Comput. Electr. Eng., pp. 196-200, 2013.

[17] N. Y. Dai and M. C. Wong, "Design considerations of coupling inductance for active power filters," in 2011 6th IEEE Conference on Industrial Electronics and Applications, 2011, pp. 1370-1375.

[18] Y. Zhao, N. Dai, and BaoAn, "Application of three-phase modular multilevel converter (MMC) in co-phase traction power supply system," in 2014 IEEE Conference and Expo Transportation Electrification AsiaPacific (ITEC Asia-Pacific), 2014, pp. 1-6.

[19] A. Marzoughi, R. Burgos, D. Boroyevich, and Y. Xue, "Analysis of capacitor voltage ripple minimization in modular multilevel converter based on average model," in 2015 IEEE 16th Workshop on Control and Modeling for Power Electronics (COMPEL), 2015, pp. 1-7. 